\title{
Belgeo
}

Revue belge de géographie

3-4 | 2011

Human mobility and housing market during a period of global recession - Miscellaneous

\section{Thèses de doctorat et mémoires de licence et de maîtrise en géographie (2007-2011) / Doctoraats- en licentie- en masterverhandelingen geografie (2007-2011)}

\section{(2) OpenEdition Journals}

Édition électronique

URL : http://journals.openedition.org/belgeo/6326

DOl : $10.4000 /$ belgeo.6326

ISSN : 2294-9135

Éditeur :

National Committee of Geography of Belgium, Société Royale Belge de Géographie

\section{Édition imprimée}

Date de publication : 31 décembre 2011

Pagination : 221-252

ISSN : 1377-2368

Référence électronique

«Thèses de doctorat et mémoires de licence et de maîtrise en géographie (2007-2011) / Doctoraatsen licentie- en masterverhandelingen geografie (2007-2011) », Belgeo [En ligne], 3-4 | 2011, mis en ligne le 15 décembre 2012, consulté le 20 avril 2019. URL : http://journals.openedition.org/ belgeo/6326; DOI : 10.4000/belgeo.6326

Ce document a été généré automatiquement le 20 avril 2019.

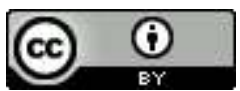

Belgeo est mis à disposition selon les termes de la licence Creative Commons Attribution 4.0 International. 


\section{Thèses de doctorat et mémoires de} licence et de maîtrise en géographie (2007-2011) / Doctoraats- en licentie- en masterverhandelingen geografie (2007-2011)

\section{NOTE DE L'ÉDITEUR}

Cette liste fait suite à celle publiée dans Belgeo, 2006, 4, pp. 473-492.

Deze lijst is een vervolg van deze gepubliceerd in Belgeo, 2006, 4, pp. 473-492.

\section{Thèses de doctorat - Doctoraatsverhandelingen}

- FUNDP (Namur)

\section{8}

2 Denil Barbara, Community-based geographical information system to assist cities in adressing food security issue through urban and periurban agriculture. The case of Bacolod City, Philippines

$3 \quad 2010$

4 Daix Nicolas, Internal rural migration and marginality: the case of Agusan del Sur, Philippines

- Katholieke Universiteit Leuven 
De Baets Sarah, The effect of plant roots on rill and gully erosion: application to a Mediterranean plant system

De Laet Veronique, Evolution and reconstruction of the geo-archaeological landscape in the territory of Sagalassos (SW Turkey): integration of geomorphic, GIS and remote sensing methods

De Maesschalck Filip, Stadsgewestvorming en electoraal-geografische dynamiek. Een onderzoek in Brussel en Antwerpen

Knapen Anke, Spatial and temporal variability of the erosion resistance of loess-derived topsoils during concentrated runoff

Lievois Els, De geografie van het toerisme in de stad: bepaling van toeristiciteitsindicatoren en methodiek voor interactie-analyse

Loopmans Maarten, Urban governance, neighbourhoods and organised residents: resident mobilisation and urban policies in Antwerp, Belgium

Molina Armando, Land use change, runoff and erosion in a degraded catchment in the Andes: determining pathways of degradation and recovery

Peeters Iris, Spatial Modelling of Sediment Redistribution Patterns on a Millennial Time Scale

Rommens Tom, Holocene sediment dynamics in a small river catchment in Central Belgium

Vu Kim Chi, Land use change in the Suoi Moi catchment, Vietnam: disentangling the role of natural and cultural factors

\section{8}

Leys Annemie, Hydrological and erosion aspects of conservation agriculture

Newton Caroline, Social Housing, Urban Policy and Social Capital: Spatial Interrelations in a Third World Context (Cape Town)

\section{9}

Demuzere Mattthias, A downscaling approach for air quality at a mid-latitude site using circulation patterns and surface meteorology

De Vente Joris, Soil erosion and sediment yield in Mediterranean Geoecosystems

Lauwaet Dirk, The influence of land use changes on precipitation in the Sahel

Notebaert Bastiaan, Sensitivity of river systems to human actions and climatic events across different environments: a Holocene perspective

Smets Toon, Effectiveness of biological geotextiles in controlling soil erosion by water at a range of spatial scales

Zenebe Amanuel, Assessment of spatial and temporal variability of river discharge, sediment yield and sediment-fixed nutrient export in Geba river catchment, Northern Ethiopia

\section{0}

Poelmans Lien, Modelling urban expansion and its hydrological impacts

Van Dessel Wim, Evaluation of land cover change models in rural areas in central and Eastern Europe

Van Weverbergh Kwinten, Modelling of extreme precipitation in Belgium 
2011

Dusar Bert, Late Holocene sediment dynamics in a Mediterranean mountain environment Langhans Christoph, The effect of rainfall intensity and water depth on the scaledependency of infiltration on a hillslope

Meeus Bruno, Migrant workers and postsocialism. A social reproduction perspective to work migration from North-East Romania

Schuermans Nick, Anxieties, identities and spatialities: ambivalent geographies of encounter in Cape Town and Flanders

Stuyck Karen, Een sociale geografie van remittancespraktijken : Transfers van België naar Senegal als case-studie

Van den Putte An, Application of conservation tillage in European cultivated landscapes: possibilities and limitations

Verachtert Els, Soil piping in a temperate humid climate. The Flemish Ardennes (Belgium)

Wang Zhengang, Towards a better understanding of the fate of carbon mobilized by erosion

\section{- Universiteit Gent}

\section{7}

Vanderstraete Tony, The Use of Remote Sensing for Coral Reef Mapping in Support of Integrated Coastal Zone Management - A Case Study in the NW Red Sea

Van Eetvelde Veerle, Van geografische strekenkaart tot landschapsdatabank. Gebruik van GIS, informatietheorie en landschapsmetrieken voor het karakteriseren van de landschappen, toegepast in België

\section{8}

Bogaert Peter, A Qualitative Calculus for Moving Point Objects Constrained by Networks

(Een Kwalitatieve Calculus voor bewegende puntobjecten gebonden door netwerken)

Ghysels Gunther, Bijdrage tot de studie van de kenmerken, de genese en de datering van periglaciale polygonale wigstructuren in België

6 Van der Putten Nathalie, Post-glacial palaeoecology and palaeoclimatology in the subAntarctic

\section{9}

Deprez Sarah, Geoarchaeological study of the natural resources in the territory of the Roman town of Ammaia (Northeastern Alentejo, Portugal). Case studies on water supply, granite building stone quarrying and opencast gold mining

\section{0}

Devriendt Lomme, Nodal points in the space of informational flows: an empirical analysis of

transnational urban networks based on Internet and air traffic flows 

Protected Areas: Implications for Management and Conservation, Democratic Republic of Congo

Neutens Tijs, Space, Time and Accessibility. Analysing Human Activities and travel possibilities from a Time-Geographic Perspective

Sevenant Marjanne, Variation in landscape perception and preference. Experiences from case studies in rural and urban landscapes observed by different groups of respondents

Van Acker Veronique, Spatial and social variations in travel behaviour. Incorporating lifestyles and attitudes into travel behaviour-land use interaction research

Van Bogaert Rik, Recent treeline dynamics in sub-Arctic Sweden, a multi-disciplinary

landscape assessment

Veraverbeke Sander, Assessing fire/burn severity using spaceborne spectral indices

2011

Bassens David, Emerging Nodes on 'Alternative’ World City Networks: The Case of Islamic Financial Services

Boussauw Kobe, Aspects of spatial proximity and sustainable travel behaviour in Flanders: A quantitative approach

2 Delafontaine Matthias, Modelling and analysing moving objects and travelling subjects. Bridging theory and practice

Hassens Heidi, The geography of contemporary urban systems at various spatial scales An empirical analysis based on information flows in the advanced producer services sector

Kellens Wim, Analysis, Perception and Communication of Coastal Flood Risks. Examining objective and subjective risk assessment

Vanoutrive Thomas, From mobility management and multilevel modeling towards modeling mobility and multilevel management

\section{2}

Tack Frederik, Assessing 3D City Models Automatically Extracted From High Resolution spaceborne Images

\section{- Université catholique de Louvain}

\section{8}

Evrard O., Muddy floods in the Belgian loess belt : problems and solutions

Lorena R., Linking spatial patterns of land use to agents of deforestation in the Brazilian Amazon

Meerkerk A., Rainfed orchards in semi-arid environments : Retaining the water and the soil

Rowhani Ardekani P., Global interannual variability in terrestrial ecosystems : Patterns, sources, and impacts on biodiversity and human security

Stevens A., Changes in soil organic carbon at regional scales: strategies to cope with spatial variability 
Swinnen E., Vegetation dynamics in Southern Africa from NOAA-AVHRR and SPOT-VGT time series

2009

Conchedda G., Human and Environment interactions in two Mangrove Ecosystems of Senegal

Frot E., Runoff as an additional water resource in a semiarid karstic aquifer

Meyfroidt P., Forest Transition in Vietnam: Evidence, Theory and Social-Ecological Feedbacks

Goidts E., Soil organic carbon evolution at the regional scale : overcoming uncertainties and quantifying driving forces

Linard C., Spatial and integrated modelling of the transmission of vector-borne and zoonotic infections

\section{0}

Haas E., Temporary water bodies as ecological indicators in West African drylands

Obsomer V., Multiscale environmental analysis and prediction for insect vector of desease : application to malaria vectors in Southeast Asia

\section{1}

Dion E., Landscape epidemiology of foot-and-mouth disease in South Africa : a spatiallyexplicit multi-agent simulation model

Lorent H., Human-environment analysis of land degradation at the level of farms. Three case studies in Mediterranean Europe

Vandenbulcke G., Spatial Analysis of Bicycle Use and Accident Risks for Cyclists

\section{- Université de Liège}

\section{8}

Daoudi Mohamed, Analyse et prédiction de l'érosion ravinante par une approche probabiliste sur des données multisources. Cas du bassin versant de l'Oued Isser - Algérie

Devillet Guénaël, Modes de déploiement des réseaux des entreprises multi-établissements du commerce de vêtements en Belgique : vers une approche stratégique?

Niang Abdoul Jelil, Les processus morphodynamiques, indicateurs de l'état de la désertification dans le sud-ouest de la Mauritanie. Approche par analyse multisource

\section{9}

Ibannain Fatiha, Modélisation des données géographiques de référence au Maroc. Préalable à la mise en place d'une infrastructure nationale de données spatiales Lejeune Sandrine, Influence de l'ionosphère sur le positionnement différentiel par GNSS

Mai Thành Tân, Géorisques au centre septentrional du Vietnam

\section{0}

Hallot Eric, Typologie hydro-géomorphologique des cours d'eau dans l'Euregio MeuseRhin

Rixhon Gilles, Datation de l'incision quaternaire des rivières du massif ardennais par les nucléides cosmogéniques terrestres (10BE/26AL) 


\section{1}

Melnik Alexandre, La gestion des risques naturels et anthropiques dans la région du lac Baïkal : une approche spatio-temporelle

Muhindo Sahani Walere, Le contexte urbain et climatique des risques hydrologiques de la ville de Butembo (Nord-Kivu/RDC)

Trinh Hong Loan, Property Taxation and Land Use Management in Vietnam: the potential for Reform

\section{- Université Libre de Bruxelles}

\section{7}

Dobruszkes Frédéric, Géographie de la libéralisation du transport aérien passagers en Europe

Vandeburie Julien, Structures d'appropriation, conflits et ressources naturelles

2009

Van Hamme Gilles, Classes sociales et géographie des comportements politiques en Europe occidentale

\section{1}

Bauthier Isabelle, La valorisation touristique des territoires par les événements sportifs : une analyse sur le long terme. Le cas des Jeux Olympiques d'été (1984-2004)

Geilfus Nicolas-Xavier, Inorganic carbon dynamics in coastal arctic sea ice and related airice $\mathrm{CO} 2$ exchanges

1 Olmos Carlos, Gestion des ressources hydriques des villes de La Paz et d'El Alto (Bolivie): modélisation, apports glaciaires et analyse des variables

\section{- Vrije Universiteit Brussel}

\section{7}

Magosse Reinoud, Branding Brussels as the capital of Europe?

2008

Maro Pendo, Environmental change in Lesotho : an analysis of causes and consequences of land use change in the lowland region

Tysmans Daisy, Size and shape of small sedimentary particles : a proxy for the palaeo environment?

\section{1}

Van de Voorde Tim, Mapping, monitoring and modelling urban areas with mediumresolution satellite imagery. A multi-resolution approach for characterising the dynamics of urban form and function 


\section{Mémoires de licence et de maîtrise - Licentie- en masterverhandelingen}

\section{- Katholieke Universiteit Leuven}

2007

Clymans Wim, Impact of Soil and Water Conservation measures on the hydrology and sediment budget of a subtropical catchment : May Zeg Zeg (Northern Ethiopia)

Coremans Katrien, De impact van versnippering op de visuele appreciatie van het landschap

Devos Evelyne, Economische aantrekkingskracht van grensregio's. Case : West- en OostVlaamse bedrijven in Nord Pas de Calais (F)

Dusar Bert, Bijdrage tot de reconstructie van de fluviatiele geomorfologie van de Nijlvallei tijdens het Holoceen in de omgeving van Deir al-Bersha

Horemans Eline, Kiezen voor participatie bij brownfieldherontwikkeling? Case study Vlaanderen : Trefil Arbed Gentbrugge

Konings Pieter, Dorpskernverschuiving: Nederzettingstransformatie van Guigoven en Bommershoven

Kwakernaak Bert, De relatie tussen atmosferische circulatiepatronen en neerslag in België

Lambaerts Kim, Wat is de impact van de Ruimtelijke Economische Hoofdstructuur van Vlaanderen op het vestigingsmilieu voor kenniseconomie in Vlaanderen?

Lambrechts Lien, Effects of land use (changes) on river channel morphology in the Jadan catchment, Ecuador

Mattyssens Jesper, Reconstructie van historische landgebruikspatronen in het Nethenbekken

Moonen Pieter, Bio-geomorfologische interacties in schorren

Nackaerts Klaartje, Calibratie en validatie van het Clue-S landgebruiksmodel met behulp van Corine Land Cover invoerdata

Nevelsteen Kristof, Ruimtelijke analyse en voorspelling van de sedimentatie in een gecontroleerd overstromingsgebied

Peeters Jonathan, De prijsstructuren op de privéwoningmarkt in Vlaanderen en Brussel

Reynders Martin, Begroting van de Holocene alluviale sedimentopslag in de Amblève

Steegmans Tristan, Evaluatie van wolken- en neerslagprocessen in het Lokal Modell

Van De Putte Tom, De oppervlaktetemperatuur en klimatologie van de nieuwe Belgische basis in Antarctica : Een studie met model en satelliet data

Van Kerckhoven Stijn, Assessment of sediment production and sediment yield in a large basin affected by El Nino : the Catamayo-Chira basin, Ecuador-Peru

Vandendael Michael, Erosie- en depositiepatronen in de vallei van de Béoux: Rol van landgebruiksveranderingen 
Vanfraechem Ellen, Toepassingsmogelijkheden van elektrische resistiviteit in geomorfologisch en archeologisch onderzoek

Vanmaercke Matthias, Magnitude and Dynamics of Runoff and Sediment Transport in the Geba River Catchment, Northern Ethiopia

Verbeek Eefje, Het bestemmingsimago van Haspengouw. Case studie Sint Truiden

Vervenne Antonie, Attitudes en ruimtegebruik van bewoners in een gestudentificeerde stad. Case study Leuven

Vissenaekens Natasja, Bijdrage tot de begroting van de alluviale sedimentopslag in de Bügdüzvallei (Sagalassos, $\mathrm{SW}+\mathrm{C} 41 \mathrm{~W}$-Turkije)

Wemans Katelijne, Mechanische effecten van Mediterrane plantenstengels en -wortels op de site-erosieweerstand tijdens geul- en ravijnvorming

\section{9}

Akkermans Tom, Regime-afhankelijk modelevaluatie van parameters gerelateerd aan wolk- en neerslagprocessen in het COSMO-model

Claes Martijn, Modelgebaseerde landgebruikreconstructies in het territorium van de klassieke stad Sagalassos (Turkije)

Clybouw Jennifer, Land use and land cover dynamics in Lafayette County (Mississippi, USA) for the period 1830-2008 and its impact on sediment dynamics

Devis Annemarie, The reproduction of Föhnwinds and low level jet streams at the leeside of the Antarctic Peninsula in a high resolution atmospheric model

Goffin Jasper, Welke standplaats en bosontwikkelingsfactoren hebben een invloed op de houtkwaliteit bij zomereik (Quercus robur L.)

Hermy Jozefien, Long-term and historical sediment dynamics in a mountainous Mediterranean catchment : a case-study on the Büğdüz river catchment (SW Turkey)

Kusters Heleen, De Sahara van Lommel. Temporele reconstructie van de ruimtelijke dynamiek van het stuifzandgebied en de reacties van de lokale gemeenschap hierop

Peeters Ingmar, De ontwikkeling van de biologische wijnbouw in de Côtes du Ventoux

Van Dyck Ward, Changes in river morphology and stream valley sedimentation in Northern Mississippi (USA) for the period 1830-2008

Van Gils Liselotte, Possibilities and constraints for regional development by (heritage) tourism in rural China. Case : the World Heritage Site of Longxian Village in Qingtian County

Van Hecken Michiel, Analyse van de fluviatiele dynamiek in het Dijlebekken (1969-2008) op basis van veldgegevens en modellering

Van Hemel Nick, Landgebruikveranderingen en waterverbruik in Mediterrane kustgebieden : een gevalstudie in Baix Emporda (Catalonië, Spanje)

Vanden Broucke Sam, De relatie tussen de grootschalige atmosferische circulatie en de oppervlaktewind in een studiegebied rond België : een studie met een classificatie van circulatieweertypes

Vanderlinden Joke, Peri-urbane landbouw rond Brussel

Vermeiren Karolien, A case study of gentrification in Woodstock, Cape Town : a dividing process 
2010

Beckers Veronique, Analysis of the relation between NDVI and precipitation in the Andes mountain range based on low resolution satellite data

Bergmans Tine, Struggles over urban space : case-study of stateled urban restructuring and squatters' struggles over urban space

Boets Bram, De Belgische kust : complementariteit of spanningsveld tussen toerisme en stedelijkheid?

Broothaerts Nils, Spatial and temporal patterns, causes and consequences of landslides in the Gilgel Gibe catchment, Ethiopia

Dewulf Bart, Verticale extrapolatie van windsnelheidsmetingen naar de ashoogte van windturbines voor drie sites in Europa

Druyts Gert, Analyse van stedelijke morfologie en landgebruik met behulp van remote sensing en ruimtelijke maten, een casetudie op Istanbul

Duhayon Jan, Analyse van de perceptie van verkeerslandschappen door jonge kinderen door middel van eye-tracking

Enkels Koen, The medium-term impact of a catastrophic landslide dam on the basin sediment yield

Gaens Joris, De invloed van landbouwstructuren en -productiviteit op de plattelandsontwikkeling in Vlaanderen

Haex Jill, De rol van landbouwterrassen in het fluviatiele sedimentbudget van de Bügdüzvallei, SW Turkije

Herremans Kristof, Experimental assessment of runoff and sediment trapping effectiveness of vegetative filter strips in the riparian zone of lake Victoria

Jena Tuur, Onderzoek naar het suburbanisatieproces te Overijse, Een vergelijkende casestudy : Jezus-Eik, Maleizen en Terlanen

Kelchtermans Nele, Haalbaarheid van de onderzoekscompetentie wetenschappen in de 3 ${ }^{\text {de }}$ graad ASO en meerwaarde van Google Earth bij excursievoorbereiding

Maes Marieke, The combined effect of the post-socialist transition and the shrinkage of the life conditions in Ostrava (Czech Republic) : the case-study of Hrusov

Noppe Bart, De tweedehands automarkt in Vlaanderen en haar relatie met Oost-Europa via rechtstreekse verkoop

Pareijn Marlon, Historische analyse van de ruimtelijke spreiding van de voetbalclubs in Vlaanderen met als case-study de Midden Kempen

Segers Jenny, Karakterisering van de stedelijke morfologie en het landgebruik aan de hand van remote sensing data en ruimtelijke maten, toegepast op de greater Dublin Area

Severi Bart, Implementatie van landschapsappreciatiemodellen in een GIS-omgeving

Steelandt Annelies, De aanbodzijde van het oorlogstoerisme en de implementatie van een strategisch beleidsplan voor toerisme en recreatie, Case study de Westhoek

Valgaeren Jeroen, Geel en de Gheelodroom: een stadsproject met het oog op een begeleide gematigde verstedelijking

Van Asbroeck Mathijs, Studie tot het bepalen van de optimale route over Oost-Antarctica in het kader van de "Antarctic matters expedition 2011-2012" 
Van Bruyssel Simon, Interactie tussen demografie en landgebruik in vijf studiegebieden in Tigray, Ethiopië (1994-2009)

Vandeperre Joeri, Relative importance of small and large reservoirs on the sediment export from a catchment

Van Turnhout Pieter, Mechanismen van ontbossing in het stroomgebied van de Gilgel Gibe, Zuidwest Ethiopië

Vereycken Koen, De impact van landgebruiksveranderingen op overstromingsrisico's : een gevalstudie in het bekken van de Molenbeek (Z-O Leuven)

2011

Adiyia Bright, Detection and typology of urban agriculture in developing countries, case study : Kampala (Uganda)

Beckers Pieter, Ensemble Classification of Natura 2000 Heathland habitats using hyperspectral imagery

Beringhs Katia, Change detection in een berggebied: vergroening en degradatie van de Andes met spot-vegetation en NOAA-AVHRR GIMMS (1999-2006)

Briké Frederik, Social exclusion in desindustrialising regions: the case of southern Luxembourg

Cockx Kasper, Het bouwblok en "Quality of life": een typologie van de Antwerpse leefomgeving op basis van een "sliding neighbourhood"-benadering

De Brue Hanne, Hoe werden rotsblokken naar het Kempisch plateau getransporteerd?

De Bruyn Tom, Political representation of minorities in the city of Cape Town

De Petter Benjamin, Ruimtelijke analyse van ongevallenconcentraties op Vlaamse snelwegen: identificatie van gevaarlijke segmenten op basis van geregistreerde letselongevallen (2003-2007)

De Witte Pieter, Intermodaal transport en intermodale transportplatformen in Vlaanderen en Nederland

Geeraert Naomi, Modelling the front variations of the Morteratsch glacier, Switzerland

Geerts Sandra, Detectie en modelleren van stedelijke groei in Caïro en studie van de impact van deze groei op het cultureel erfgoed

Gérardy Elisabeth, Analyse van het huidig regionaal-economisch beleid van een Henegouwse intercommunale (IDEA) volgens de theorie van de Learning Region

Ghyselen Rob, Grensoverschrijdende verschillen als katalysator voor grensarbeid en migratie. Gevalstudies voor de Belgisch-Franse en Frans-Luxemburgs-Belgische grensstreek

Gorssen Ben, Ruimtelijke dynamiek in twin cities, Gevalstudie Hasselt-Genk : realiteit of utopie?

Hamels Koen, Thresholds and impacts of gully erosion in South Western Uganda

Janssens Evelien, Soil organic carbon stocks and distribution in a subtropical climate Arvorezinha, Brazil. A comparison of quantitative analysis techniques for soil organic carbon

Meers Dries, Rekeningrijden in Brussel: Stockholms good practice en de optimale schaalkeuze 
Pardon Adriaan, Kenmerken van diffusie van een innovatie : huishoudens met zonne panelen in Leuven

Petrlic Kristien, Spatial patterns of vegetation in a humid badland area. Case study : inner Pyrenean depression

Ryken Nick, Assessment of spatial and temporal variability in stream flow and sediment yield in the upper Rwizi catchment, Southwest Uganda

Sieprath Kristel, Integration of remote sensing and palynology to study the land cover in the territory of Sagalassos

Thiery Wim, Modelling surface and snowdrift sublimation at the Belgian Antarctic station Princess Elisabeth

Thijs Joris, De woningmarkt en socio-ruimtelijke ongelijkheden. Een vergelijkende studie : Antwerpen, Rotterdam en Hamburg

Vandepoel Melissa, Impact van het genderaspect op het ruimtegebruik door bezoekers in steden. Case study : Brussel

Vanesch Jolina, Het voorspellen van de onverstoorde sedimentflux van kleine en middelgrote riviersystemen in Europa

Vercammen Jochen, Towards a landslide susceptibility map for the Gilgel Gibe Catchment (Southern Ethiopia)

4 Vercauteren Alexandra, The effects of conservation tillage on the soil : a comparison between the root density and the bulk density of winter wheat, potato and maize under conventional tillage and reduced tillage

Verlinden Wim, Agent based modelling of pre-European deforestation on islands in the Pacific

Willemsen Evelien, Sociaal kapitaal in de paardensector

\section{- Universiteit Gent}

\section{7}

\section{Geografie}

Arnout Bert, Een ruimtelijke analyse van steden met meerdere luchthavens

Claerman Yannick, De invloed van de studenten op de structuur en morfologie van de stad Gent,

De Baere Sophie, Semiologie en randgegevens op toeristische kaarten in Vlaanderen

De Bock Veerle, Kwantitatieve reconstructie van veranderingen in de paleo-productiviteit van Antarctische kustmeren (Beak Island, Antarctisch Schiereiland)

De Bruyne Jeroen, Verplaatsingspatronen van 65+-ers in het stadsgewest Gent

De Bus Tijs, Het gebruik van thermale banden (Landsat en ASTER) in functie van het voorkomen van permafrost in het Altaigebergte (Dzahzator) vallei, Siberië, Rusland

De Cock Charl, Fysisch milieu en ontwikkeling. Het Noord-Atlantisch orkaanseizoen 2005. De gevolgen voor de Golf van Mexico

Depret Kevin, Wat is het maatschappelijk draagvlak van autodelen? 

model Zeegebied regio Roeselare Europa energiebronnen

De Sutter Nathalie, Sauga Landslide in Western Estonia as expressed in digital terrain

De Troch Robin, Wonen boven winkels : een empirische toepassing voor Gent en Aalst

Gillijns Koen, Geomorfologische studie van de Huerva-vallei tussen Muel en Mezalocha ; Z van Zaragoza, Aragón, Spanje

Hanssens Heidi, Studie en evaluatie van een aantal meetinstrumenten voor sociaaleconomische ontwikkeling

Hollevoet Nicolaas, Studie van de gletsjers- en gletsjermerenpositie in het Altaï-geberte (zuidelijke Chuonbergketen, Rusland) gebaseerd op Landsat-data tussen 1975 en 2001

Lahousse Sofie, Analyse van hellingen en valleibodems in het centrale deel van de Ebrodepressie (Zaragoza, Spanje)

Langerock Anneleen, Bijdrage tot de studie van travertijn-afzettingen in het Dode

Lebrun Delphine, Geomorfologische en geobotanische analyse in de omgeving van Fuendetodos (Zaragoza, Spanje)

Meirlaen Koen, Diversiteit in Scouting. Op zoek naar sociaal-ruimtelijke patronen in Gent

Mennes Filip, Indian-Atlantic Water exchange during the last 350000 years

Mulkens Eline, De ruimtelijke socio-economische structuur van de stad Antwerpen, nader geanalyseerd: een empirische studie

Reunes Nick, Vergelijkende studie van IKONOS en QuickBird beelden voor stedelijke DEM's (toegepast op Luik)

Rogolle Christophe, De ruimtelijk-economische dynamiek van de groentesector in de

Schaeck An, De relatie tussen typisch toeristische luchthavensteden en wereldsteden: een empirische analyse

Spillebeen Carlo, Een netwerkanalytische benadering van de wereld-stedenstructuur in

Tyberghein Lennert, Ruimte voor energie in Vlaanderen. Nadruk op hernieuwbare

Van Daele Jelle, Studie van hoge oppervlaktetemperaturen aan de hand van ASTER- en MODIS-beelden in het Tassili gebergte (Algerije)

Van de Kerckhove Ruben, Geomorfologische studie van Ploegsteert en omgeving aan de hand van terreinonderzoek

Van de Moortel Frauke, Reconstructie van litorale paleoproductiviteit en zeeijs-dynamiek in relatie tot Holocene klimaatsveranderingen op Beak Island (Antarctisch Schiereiland)

Van Nieuwenhuyze Katrien, Studie van de problematiek van het afsmelten van de gletsjers van de Kilimanjaro, Mount Kenya en het Ruwenzori gebergte (Oost-Afrika)

Van Nieuwenhuyze Wim, Paleolimnologische reconstructie van Mid- en Laat-Holocene klimaatveranderingen in South Georgia (Sub-Antarctica)

Van Steenbrugge Sofie, Het spreidingsbeleid voor asielzoekers in België. Onderzoek naar de factoren in de woonplaatskeuze van een asielzoeker 

behulp van GIS Altaï gebergte Marchande Couffo (Benin)

Veraverbeke Sander, Studie van Landschappelijke Veranderingen en Landdegradatie aan de hand van Teledetectie en GIS op het eiland Chios, Griekenland

Verbeek Thomas, Impact vanuit de Nederlandse grensregio op de ruimtelijke dynamiek in het buitengebied van de Noorderkempen. Residentiële inwijking - grensoverschrijdende wegverbinding Turnhout-Tilburg

Verleye Thomas, Het Holocene paleomilieu van de zuidwestelijke Zwarte Zee: een reconstructie aan de hand van dinoflagellatencysten en andere palynomorfen

\section{Landmeetkunde}

Blindeman Lieve, Wetenschappelijke inventarisatie van topografische toestellen behorend tot het patrimonium van het Museum voor de Geschiedenis van de Wetenschappen van de Universiteit Gent

Bral Lander, Vergelijkend onderzoek van de nauwkeurigheid van laserscanning, fotogrammetrie en totaalstation bij het opmeten van objecten in 3D

Callens Barbara, Cartografie van de ruimtelijke planning in Nederland

Couge Dorine, Ruimtelijk management van het provinciaal domein "De Gavers" op grondgebied Geraardsbergen

De Baets Koen, Opstellen van een knelpuntenkaart voor een fietsroutenetwerk met

Demuytere Joris, Evaluatie van debietmetingen aan de hand van chemische methodes op rivieren : praktische aspecten

De Ryck Marijke, De virtuele reconstructie van de "Porte de Landelies" (Thuin) met behulpvan fotogrammetrie

De Schryder Els, Analyse van hightech plaatsbepalingsapparatuur ter opsporing van ondergrondse nutsleidingen voor het voorkomen van graafschade

De Wit Bart, Kaartreeksen, coördinaat-systemen en de kaartindeling van de grootschalige officiële topografische kaarten van België vanaf de eerste basis-kaart tot heden

Dossche Dries, Fotogrammetrische restitutie van Corona KH-4B beelden voor de kartering van Koerganen en andere archeologische structuren in het Kazachse gedeelte van het

Dossche Koen, Studie van het generalisatieproces van de Kabinetskaart tot de Carte

Jonckheere Maximimiliaan, Evaluatie van debietmetingen aan de hand van chemische methodes op rivieren : theoretische aspecten

Kindermans Pieter, Inventarisatie, georeferentie en nauwkeurigheidstudie van topografische data en cartografische gegevens van de Kemmelberg

Lambrecht Robbe, Opmetingen en kartering van natte landbouwgronden in de Mono-

Nuttens Timothy, De virtuele reconstructie van de Acropolismuren van Titani (Peloponnesos, Griekenland), met behulp van fotogrammetrie

Pernet Koen, Studie van de gletsjerbe-wegingen in het Mont Blanc Massief tussen 1975 en 2004 aan de hand van Landsat en ASTER-beelden

Verhees Elke, Vergelijkend experimenteel onderzoek van GPS-apparatuur op basis van het ontwerp van de nieuwe ISO-norm 17123-8 
Wallaeys Nils, Onderzoek van de invloed van lenskalibratie op de nauwkeurigheid van fotogrammetrische gegevensacquisitie

2008

\section{Geografie}

De Bruyne Jeroen, Activiteitenspatronen van 65+-ers in het stadsgewest Gent

Kevin De Paepe, Bijdrage tot de geomorfologische kennis van de boven Schelde van de regio Gavere - Oosterzele (kaartblad 22/5-6; 1/20.000)

Bob D'haeseleer, Democratie in het wereld-systeem

\section{Landmeetkunde}

Absil Kevin, Studie over de mogelijkheden van het gebruik van een terrestrische fotogrammetrische camera in de luchtfotografie

Maes Tim, Onderzoek naar verplaatsingsgedrag in Sint-Niklaas a.d.h.v. een decompositioneel multi-attribuut preferentiemodel

2009

\section{Geografie}

Burggraeve Steve, Toepassing van een kwalitatieve trajectcalculus op een complexe verkeerssituatie

De Blieck Fréderic, De invloed van de klimaatverandering op het moessonregime; met nadruk op de Aziatische moessons

Decoene Kristof, Het Europees Parlement : kiezen tussen symboliek en efficiëntie

De Craene Valerie, Geopolitiek van de Verenigde Staten aan de hand van cijfers over de ontwikkelingshulp

de Keyzer Sarah, De toenemende vergrijzing en haar effect op wonen

De Meersman Bart, Risico in beeld : inventarisatie van het risicobeleid in Vlaanderen

de Mûelenaere Stephanie, Land use and cover changes in the Ethiopian highlands; landsat data analysis using contemporaneous ground photographs for calibration

De Roo Natascha, Fysisch milieu en ontwikkeling: impact van de Alqueva stuwdam in Portugal

De Tavernier Klaas, Integratie van de historische dimensie in een landschapstypologie door middel van GIS, toegepast op de landschappen rond Brugge

De Vos Anneleen, Dynamiek van het wereldstedennetwerk: longitudinale analyse 2000-2008 aan de hand van GaWC-data

De Weser Stefanie, De onafhankelijkheid van Kosovo en de achterliggende geopolitiek

De Winter Jonas, Klimaat en ontbossing in het Amazonegebied

Erauw Pieter, Supertrucks : oplossing voor de toenemende vraag naar vrachtvervoer?

Jongepier Iason, Historisch-geografisch onderzoek van de Moervaartdepressie en omgeving. Een studie aan de hand van historische kaarten tussen de $13^{\text {de }}$ en de $18^{\text {de }}$ eeuw en literatuur

5 Keerstock Melissa, Studie van de sociaal-economische differentiatie in functie van de fysische geografie in de stad Napels 

gang)

Lauwaert Jasmin, Klimaatverandering in Mexico : impact op het leven van Mexicanen en adaptaties van de Mexicaanse overheid

Malschaert Jelle, Fysisch Milieu en Ontwikkeling. Impact van de Drieklovendam (China)

Meire Ellen, Mapping land use and cover in the North Ethiopian Highlands since 1868 using warped terrestrial photographs

Neyens Karen, Al-Idrisi : onderzoek naar zijn bronnen, een wiskundige benadering van de regio rond België, en de invloed van Ptolemaeus

Pirlet Pieterjan, De randstad binnen de regulatietheorie (een herschalingsproces aan de

Plasschaert Katrien, Concentratie in netwerken: toepassing van verschillende concentratie-indices op luchtvaartnetwerken

Rohaert Daniël, Bijdrage tot de studie van de geomorfologie en geoarcheologie in de provincie Cadiz (Spanje)

Santens Koen, Landschapskundige impact van bodemerosie en erosiebestrijdingmaatregelen in de Vlaamse Ardennen. Case studie van de gemeente Zwalm

Stragier Frederic, Kritische analyse van de legende van de Kabinetskaart van Ferraris

Van Beveren Tim, De landschapsdynamiek van de ankerplaats "De Merelbeekse Bossen" en omgeving. Analyse vanaf de $18^{\text {de }}$ eeuw tot heden door middel van tijd-ruimte composieten en landschapsmetrieken

Vandekerckhove Frederik, Onderzoek naar landschapsdynamiek en krachten van landschapsveranderingen in Vlaanderen, een vergelijkende studie van drie nederzettingen (Zillebeke - Nokere - Hoegaarden)

Van De Vijver Elien, De invloed van transportinfrastructuren op het landschap tussen Gent en Aalst vanaf eind $18^{\text {de }}$ eeuw

Van Pottelberge Helena, Postevaluatie van project-MER's voor de discipline monumenten en landschappen. Gevalstudies in de Gentse Kanaalzone

Verbestel Katleen, De evolutie van het duinoppervlak in de Westkust. Gebruik van landschapsmetrieken voor de analyse van ruimtelijke en temporele veranderingen

Vercauteren Evelyne, Vertuining. Onderzoek naar het voorkomen in, en het effect op de stedelijke en agrarische ruimten. Gevalstudies : Temse - Kruibeke

Vermeersch Dominiek, Geomorfologische dynamiek van het ecosysteem "terril". Onderzoek van vijf terrils in België

Versichele Mathias, Implementatie in Java van een praktische tool voor de berekening en visualisatie van individuele en gemeenschappelijke bereikbaarheid

\section{Landmeetkunde}

Capelle Ruben, Analyse van methoden voor de uitvoering en verwerking van deformatiemetingen in tunnels

De Wilde Philippe, Ontwerp van een veldvalidatietest voor terrestrische laserscanning conform ISO-17123

Emonts-Gast Lieven, Nauwkeurigheid bij calibratie van een kraanpositioneringssysteem aan de hand van een sensitiviteitsanalyse 
2010

\section{Geografie} landschap op Vlaanderen de loep Vlaanderen risicoanalyse data

Serruys Eline, Risicoanalyse van de hogedrempel Seveso-inrichtingen : case studie in de Gentse kanaalzone aan de hand van GIS

Stal Cornelis, LIDAR : basisconcepten en toepassingen

Vanclooster Ann, 3D indoor navigatie van City GML

Vandenbulcke Annelies, Vergelijkende studie van softwarepakketten voor de verwerving van laserscandata

Van Dosselaer Jonas, Inertiële navigatiesystemen met inbegrip van de integratie met satellietplaatsbe-palingssystemen

Aerts Esther, Neoliberale stedelijke ontwikkelingsprojecten in "het Zuiden" : een politiek economische anlayse van de V\&A Waterfront in Kaapstad

Alleman Tim, Nationaal Olympisch succes. Analyse van de voorspelbaarheid

Baker Kevin, Studie van bodembedekking van een (sub)alpien landschap aan de hand van teledetectiedata en GIS (Altajgebergte, Rusland)

Broidioi Silke, Land use and cover changes in three small catchments in Ethiopia, with special focus on related soil fertility

Cant Patty, Onderzoek naar fysische sedimentkarakteristieken van de Demer en haar bijrivieren : Sedimentatieproeven in natuurlijk rivierwater

De Coninck Boudewijn, Neutrale modellen binnen de landschapsecologie : Evaluatie en toepassingsmogelijkheden van het "Masked Constraint Algorithm" in een verstedelijkt

De Cremer Joeri, Een analyse van de Vlaamse maatschapppelijke ruimte in het licht van de geografie van het secundiar onderwijs

Delaere Thibaut, Gebruik van viewshedanalyse voor landschapskarakterisering toegepast

De Leersnyder Sander, Een nieuw type nationalisme? Het Kosovaarse nationalisme onder

De Vos Jonas, De invloed van de residentiële mismatch op het verplaatsingsgedrag in

De Waele Arnout, Rurale bewoning in voormige kasselrij Oudenaarde: Analyse en typologie van rurale nederzettingen en evolutie van bewoningspatroon

D'hoedt Mattijs, Duurzame Europese intermodale logistieke netwerken met integratie van de Motorways of the Sea

Diependaele Stijn, Grondverschuivingen op terrils in België: geografische expliciete

Dupont Lien, Effecten van potentiële klimaatverandering op het landschap op lokale schaal aan de hand van case studies in het Denderbekken

Good Pieter, The regionalization of Africa : delineating Africa's subregions using airline 
Hendrickx Marijn, Een methodologisch onderzoek naar het gebruik van beelden genomen vanuit een microdrone voor fotogrammetrische documentatie. Toegepast op een Scytische kurganenrij in Tuekta, Altaï, Zuid-Siberië

Ingelbrecht Tijs, De integratie van voorspellingen van landgebruik in een risicoanalyse van overstromingen in Vlaanderen

Jacob Miro, Cropping systems in the Tigray highlands (North Ethiopia) as related to spatiotemporal variability of rainfall

Jacques Annelies, Paleolimnologische reconstructie van klimaatveranderingen in Lützow Holm Bay, Oost-Antarctica

Lapon Lieselot, Etniciteit in Ghana : een samenleving zonder conflicten?

Lebrun Gertjan, Vraag en aanbod van recreatie in de randstedelijke zone van Gent. Analyse van de recreatieve mogelijkheden binnen de groenpool : Gentbrugse meersen en Damvallei

Michels Nicolas, Europese Besluitvorming Politieke problemen omtrent het Europees klimaat- en energiepakket

Pontzeele Jolien, Invloed van beverdammen op de hydrologie van de Oostelijke Ourthe, met verdere focus op het dammenstelsel van de Chevral

Scholiers Nelles, Development of gully networks and volumes since 1965 in the May Mekdan catchment (Northern Ethiopian highlands)

Storme Tom, Ontwikkeling van een methode voor monitoring van landschappelijk erfgoed in Vlaanderen

Van Damme Liesbeth, Het bebouwde landschap: Karakterisering en vergelijking van functionele en morfologische verstedelijking van Aalst en Dendermonde

Van Den Branden Jeroen, Land use changes in Montenegro in the $20^{\text {th }}$ century as derived from repeat photography and hydrological response

Van Den Broeck Bram, Migratie van homoseksuelen van platteland naar de stad. Vlaanderen : een case study

Vandenbroele Lies, Het landschap als laatste getuige van de Grote Oorlog in de Westhoek : Militaire luchtfotografie als bron van landschapsonderzoek

Vervust Soetkin, Een onderzoek naar de invloed van geomorfologie op Romeinse systemen van landverdeling: Een case study in het territorium van de Romeinse stad Trea in de Potenzavallei (De Marken, Italië)

Walgraeve Veerle, Stedelijke distributie in beeld via bevoorradingsprofielen. Case studie stad Gent

\section{Landmeetkunde}

Audoor Sharon, Mogelijkheden van mobile mapping voor GRB-toepassingen

Belien Alexander, De bruikbaarheid van semantische 3D modellen voor de voorstelling van archeologische sites : toepassingen op de Sint-Baafsabdij, Gent

Danneels Eveline, Analyse van diverse kwaliteitslabels voor schattingen van onroerend goed op nationaal en internationaal vlak in Europa

Gagelmans Niels, Multitemporele analyse van het Grenspark Kalmthoutse Heide De Zoom aan de hand van hoge resolutie satellietbeelden 
2011

\section{Geografie} Gentse Feesten

Janssens Bram, Combinatie van fotogrammetrie en laserscanning voor de documentatie van het interieur van de Saint-Lambert kerk

Janssens Eva, Fotogrammetrische restitutie van Corona KH-4B beelden ter ondersteuning van de kartering van archeologische structuren (Karakol, Altaï, Siberië)

Meskens Jessica, Beïnvloedende factoren bij het meten met een laserinterferometer. Analyse van de geometrische kenmerken van een EDM-kalibratiemeetbank

Van Coster Tine, Vergelijkende analyse van lasercandata voor tunnelmetingen met behulp van softwarepakketten Trimble Realworks en Leica Cyclone

Van Hal Ward, Een methodologische studie naar het verplaatsingsgedrag van studenten met behulp van GPS

Van Londersele Bram, Captatie van de bewegingen van festivalgangers op Rock Werchter 2009 door middel van "Bluetooth Tracking"

Vanmassenhove Jessie, Fotomodellering ten behoeve van onroerend erfgoedbeheer: opstellen van een procedure

Van Nieuwenhuyse Bert, Onderzoek naar een multi-focale benadering voor fotogrammetrische documentatie toegepast op l'Eglise Saint Lambert te Bouvignes

Bogaert Wouter, Vergelijkende studie Open Source GIS : Een overzicht van de desktop mogelijkheden en een casestudie

Claeys Tim, Kan Bluetooth tracking een hulpmiddel zijn voor mobiliteitsstudies? Casestudie : Gentse Feesten 2010

Coucke Ward, De evolutie van het landschap op en rond de Kemmelberg

De Roeck Sara, Sequentieananalyse van het bewegingspatroon van de bezoekers aan de

De Roek Annelies, Een methodologie voor een driedimensionale documentatie van archeologische objecten aan de hand van fotogrammetrie

De Visscher Maarten, Sedimentatiepatronen in bevermeren en invloed op het sedimenttransport door de Chevral (Ardennen)

Du Rang Elien, Een methode voor kwantitatieve en ruimtelijke benadering van de evolutie van het trage wegennet. Toepassing voor de gemeente Herzele en ankerplaatsen

Geltmeyer Adriaan, Assessment of landscape change and visual landscape character, case studie on the Finnish island Nötö

Goethals Davy, De ontwikkeling en evolutie van nederzettingen en hun omgeving in de Peenevallei en Cassel

Ranson Elien, Historisch-geografisch onderzoek naar de relatie tussen toponiemen en het landschap in het studiegebied gelegen in de gemeenten Moerbeke, Stekene en Sinaai

Roels Jeremy, Een geografie van de "global civil society" : Internationale sportfederaties in het wereldstedennetwerk

Rotsaert Natasja, Landschappelijke analyse van de kasteelparken in de Ieperboog op regionale en lokale schaal 
Rozek Joachim, Onderzoek naar historisch topografisch instrumentarium : studie in het kader van de twintigste verjaardag van de opleiding Geomatica en Cartografie

Schockaert Tom, Gevolgen van klimaatverandering op de hydrologie in het Klein Netebekken met het WetSpa model

Stock Simon, Land use/land cover and population dynamics in North Ethiopia as derived from aerial photographs

Van Cauwenberge Frederik, Het meten van wereldsteden aan de hand van het internet: Een webinhoudsanalyse van de Google database

Van De Velde Daan, De cartografie van de neerslagschaduw bij intense regenbuien in België

Van Nieuland Jasper, De ontwikkeling van grèzes litées afzettingen in de Oesling (Groothertogdom Luxemburg)

Weyne Dieter, Het verband tussen ruimtelijke segregatie en de gevolgen van aardbevingen in Caïro : Een statistische analyse

Willen Carolien, Het gebruik van spectrale afstandwaarneming om de chronologie van lavastromen van actieve vulkanen te bepalen (Nyamulagira, Afrika)

\section{Landmeetkunde}

Algoed Marieke, Analyse van routekeuzes bij de politie in Gent

Delporte Evelien, Onderzoek naar de bruikbaarheid van fotomodellering voor de kartering van ruimtelijke objecten aan de hand van het programma Bundler

de Mûelenaere Edward, Bluetoothtracking voor marketingdoeleinden - Shoppingcenter Gent Zuid

Hauspie Jan, Nauwkeurigheid Multibeam (Foutenbudget - Scheepsgeometrie - Dynamic Draught)

Leperre Thomas, Kwaliteit van GNSS-metingen

Malfliet Joke, Onderzoek naar de bruikbaarheid van fotomodellering voor het modelleren van ruimtelijke objecten : een vergelijkende studie

Mourisse Fries, Vergelijkende analyse van software voor lenskalibratie

Van Bossche Frederik, Wireless local positioning met Bluetooth

\section{- Université catholique de Louvain}

\section{7}

Berghmans P., Les effets d'une super-éruption volcanique sur le climat mondial

Beriaux J., Influence de la gestion des forêts ardennaises sur les stocks de carbone organique des sols dans la région de la Roche-en-Ardenne

Bracaval F., Analyse de l'expansion agricole au nord de la réserve nationale du Masai Mara au Kenya

Della Faille de Leverghem A., Etude de l'impact des zones protégées sur la déforestation au Viêt-Nam

Delobel F., La déforestation et le climat des 1000 dernières années en Europe : analyse des impacts et test de différents scénarios rétrospectifs de déboisement 
De Ruette D., Impact de l'occupation du sol et d'aménagements de lutte contre les inondations sur le ruissellement et la connectivité hydrologique dans le bassin-versant de Gelinden : Apports et faiblesses du modèle expert STREAM

Detant $\mathrm{V}$., Le commerce mondial du coton : le coton comme outil de développement dans les pays africains

Dutordoir V., Etude de facteurs provoquant un ruissellement et de la connectivité dans une région semi-aride du sud-est de l'Espagne, la bassin versant de Carcavo

Fanuel J., Impact de l'histoire des changements d'utilisation du sol sur le taux de carbone dans les sols sous couvert forestier en Ardenne

Frère J., Etude de l'évolution de la population et du logement en Wallonie

410 Halbardier F., Analyse des changements de végétation à partir de séries temporelles MODIS et de données météorologiques au Grand-Duché du Luxembourg entre 2000 et 2005

411 Heyte C., Etude comparative de l'accessibilité des gares IC belges entre la semaine et le week-end

412 Jacmain C., Etude des limites inhérentes aux suivis des teneurs en carbone organique à l'échelle régionale : cas de la région limoneuse wallonne

413 Leroy M., Etude longitudinale de la fragmentation du paysage dans la région de Nkhota kota au Malawi. Analyse par télédétection

414 Le Than Nha Truc, Analyse descriptive des facteurs influençant le niveau de vie dans le milieu rural au Vietnam entre 1994 et 2001

415 Letoret A., Analyse spatiale et temporelle des inondations boueuses à Ath. Evaluation des mesures de lutte contre les inondations dans un bassin versant particulièrement touché de la commune

416 Martin C., Etude du lien entre les changements de végétation et les changements climatiques au $21^{\mathrm{e}}$ siècle

417 Noël A., Etude des aires de marché et estimation des ventes de véhicules neufs de la marque Opel en Belgique.

418 Sougnez N., Les effets de la couverture végétale sur les processus d'érosion dans les régions montagneuses du sud de l'Espagne

419 Tilman Y., Le développement économique et humain de l'Inde: sources d'inégalités régionales

421 Aubert A., L'influence de la déclivité du lieu de résidence et de l'accessibilité des services de santé sur la mortalité des personnes âgées Balthazar V., Analyse régionale du flux de sédiments dans le bassin versant du Nil Bleu Bodart M., En quoi la dégradation des sols est-elle liée au niveau socio-économique des agriculteurs de la région de Chieti Bruggeman D., La transition forestière au Vietnam: causes et perception au niveau de deux villages de la province de Bac Kan

Bruniaux A., Analyse fractale de la ville de Charleroi visant à déterminer la croissance de la ville dans le passé, corrélé à une analyse de l'âge du logement 
Duckerts B., Etude des logements et du cadre de vie à Liège, dans le but d'en comprendre les disparités spatiales et/ou sociales et comparaison des résultats obtenus à l'aide d'une analyse fractale de la ville

Guns M., Analyse de l'impact des changements d'occupation du sol sur les glissements de terrain (Equateur)

Hendrick S., Analyse diachronique des changements dans la structure spatiale des migrations : le cas des migrations inter-arrondissements en Belgique pour les années 1989 et 2006

Jones J., Relation entre accessibilité (par la route et le rail) et évolution du prix des terrains à bâtir (en Belgique, au niveau communal)

Noon Carole, La modélisation de la fonte de glace de mer arctique en 2008-2009

Van den Eynde A., Tectonique et morphologie fluviatile des rivières à graviers pavées en Ardenne belge

Antoine M., Effet d'une paramétrisation stochastique sur les cycles glaciairesinterglaciaires dans le modèle "LLN-2D"

Azzolin J., Agglomération et croissance économique. Panel de régions Nuts2 de l'UE-27 entre 1999 et 2005 Buruli dans la région du Bas-Congo, RDC

Bertoux S., Localisation des accidents de la route impliquant un cycliste. Etude des communes d'Overijse et Rixensart

Blieck G., L'accessibilité multimodale des gares exurbanisées sur les lignes européennes à grande vitesse

Decleyre N., Risques naturels en Val d'Illiez (Suisse). Analyse spatiale et temporelle des avalanches et autres dangers potentiels du versant nord-ouest des Dents du Midi

De Witte Q., Localisation optimale des futures stations de ravitaillement à hydrogène : cas d'étude pour les provinces du Brabant wallon et du Luxembourg

Dodinval J.-F., Polycentrisme et durabilité : étude dans le cadre de la ville nouvelle de Belval (G-D de Luxembourg)

Derts B., Existe-t-il un lien entre la fractalité des tissus bâtis et la perception de l'environnement immédiat à l'échelle de l'agglomération urbaine liégeoise?

Furnemont $\mathrm{C}$., Etude de l'impact à long terme des herbicides utilisés durant la guerre du Vietnam sur les forêts sud-vietnamiennes

Laschet S. Origine des fortes diminutions de l'étendue estivale de la banquise arctique observées en 2005, 2007 et 2008

Leurident N., Modélidation du rôle de la circulation océanique thermohaline dans le changement climatique abrupt survenu il y a 8200 ans

Leroy G., Peut-on prévoir l'évolution de la glace de mer dans l'Océan Austral ces 20 prochaines années? 
Linel A., Analyse de l'adaptation socio-économique des individus et ménages, face aux variabilités et changements climatiques du Sahel, pour l'ensemble du Burkina Faso de 1970 à 2000

Loicq P., Impact de la nébulosité sur la variabilité des caractéristiques de la banquise

Mairesse A., Etude des interactions entre les activités humaines, le climat et les inlandsis du Groenland et de l'Antarctique au cours des prochains siècles

Malcorps P., Activité minière au Katanga : changements d'occupation du sol et exposition humaine aux métaux

Queeckers G., Cambriolages en Belgique en 2001. Analyse des mouvements des auteurs et facteurs explicatifs du choix spatial de la cible

Renquet A., Déforestation et aléas naturels : étude de cas de mouvements de masse proches du Parc Naturel Sangay (Equateur)

Thillaye du Boullay B., Analyse des causes des sécheresses en Europe et dans le bassin méditerranéen durant les 600 dernières années : comparaison spatiale et temporelle d'un indice de sécheresse

Van de Velde Y., Evaluation à priori du cursus géographique en milieu scolaire à travers les nouvelles technologies

Verdonckt S., Développement de sol après revégétalisation de terres dégradées

Wenin M., Variabilité climatique et décisions socio-économiques des individus et des ménages au Mali entre 1970 et 2000

2011

André M., La conservation en eaux et en sols dans les Andes Equatoriennes : bilan et perspectives

Boreux M., Reconstruction des paléoclimats canadiens du dernier millénaire avec une approche combinée utilisant le modèle LOVECLIM et des reconstructions multi-proxys

Bouchoms S., Etude des changements climatiques en Chine au cours du dernier millénaire

Clapuyt F., Study of controlling factors of landslides in the Northern mountainous area of Vietnam, Sa Pa district, Vietnam : Focus on land use changes and detection on ASTER images

Cotteels C., Localisation des centres de radiothérapie en Belgique

Degryse M., Enjeux périurbains d'hier et d'aujourd'hui: trajectoires d'évolutions communales en Brabant wallon

Denis M., Analyse comparative des modèles de développement de l'Inde et de la Chine. Quels avenirs pour les géants de demain?

Dupont F., Correction des prévisions climatiques décennales sur l'Europe de l'Ouest via la technique MOS

Dufrasne R., L'urbanisme durable : une approche locale par écoquartier

Furlanetto G., Un système d'information géo-historique du réseau ferroviaire belge

Gany J., Etude de l'impact de la structure paysagère sur la présence des espèces Anopheles plumbeus, Culex pipiens, Aedes vexans et Coquilletidia richiardii en Belgique

Gauthy L., Propagation d'inondations d'origine maritime 
Godelaine S., Influence du phénomène El Nino sur la réponse hydrologique dans le bassin du Catamayo

Goulem Y., Le climat conditionne-t-il la distribution spatiale des cas humains d'encéphalite à tique en Suède entre 1986 et 2006

Hubin G., La ségrégation sociale liée aux rénovations visant à améliorer la performance énergétique des logements locatifs privés à Bruxelles en 2008

Jadin I., Comment la répartition spatiale et les caractéristiques socio-économiques des communautés ethniques sont-elles liées à la dynamique temporelle de l'utilisation du sol ces vingt dernières années dans les alentours du Parc National Hoang Lien?

Jaupart J., La mobilité des étudiants de l'Université catholique de Louvain : le choix modal des étudiants-koteurs hennuyers

Letesson G., Analyse de la construction des savoirs dans le cadre de l'enseignement de la géographie en secondaire

Malek C., Gembloux, nouveau périurbain de Bruxelles

Marescaux J., Scénarios d'impact de l'urbanisation sur les services écosystémiques à Beauvechain et Grez-Doiceau : cas du stockage de carbone organique dans le sol et de la prévention du ruissellement

Nauw D., Evolution de l'accessibilité ferroviaire en Belgique selon les planifications IC/IR de la SNCB entre 1984 et 2011

Paquet J., La réussite des migrations internationales : le point de vue des "left-behind" en Equateur

Roberti A.-S., La réussite de la migration internationale : le cas des migrants équatoriens à Bruxelles et à Valence

Shubina E., Adoption of agricultural technics in Northern Kazakhstan under the influence of Russian colonization in the end of XIX ${ }^{\text {th }}$ century

Touri S., Amélioration des prévisions saisonnières sur l'Europe du Nord

Traficanti R., Géomorphologie, topographie et formation de la chaîne bétique dans le Sud de l'Espagne

Van Roy G., Impact de la variabilité interannuelle du cycle hydrologique atmosphérique sur la glace de mer

Van Wonterghem M., Facteurs environnementaux et paysagers déterminant la fréquence de morsures de tiques : le cas des camps scouts en Wallonie

Verelst S., Urbanisation et développement. Usage de l'indicateur de développement humain pour la mise en évidence des relations existantes entre urbanisation et développement

Viroux S., La structure spatiale des petites et grandes implantations économiques dans l'agglomération bruxelloise

8 Welvaert A., Les transports aériens à bas prix : les changements géostructurels dans le triangle Francfort-Amsterdam-Paris de 1995 à 2010 et l'émergence de plates-formes spécialisées

Zeimes C., Quels sont les facteurs environnementaux qui influencent la distribution spatiale des cas humains d'hantavirus en Suède? 


\section{- Université de Liège}

\section{Géographie fondamentale}

Benoit Morgan, Le lac de Bütgenbach. Etude de la sédimentation dans la retenue par 2 approches : Sédimentologique - Hydrologique

Breuer Christophe, Analyse géographique d'une voie romaine. La section d'Arlon à Tongres

Brico Jean, Influence de l'hydrologie et de la topographie dans la localisation de l'habitat traditionnel. Etude par méthode numérique : application au Pays de Herve

Charlier Simon, Le redéveloppement de la vigne en Wallonie et en région bruxelloise

Chevau Thomas, Les marchés fonciers des terres agricoles

Dujardin Sébastien, Contribution à l'étude de la dynamique entre le tourisme et la valorisation des ressources territoriales. Étude de cas : analyse de l'offre touristique de la commune de Durbuy

Este Sébastien, Valorisation de produits locaux et développement territorial. L'exemple du fromage de Herve

Franco Bruno, Les barrages de travertin de la vallée du Hoyoux

Gillet Mathias, Évolution des polarités périphériques secondaires dans deux métropoles régionales belges. Comparaison Liège-Anvers

Guilliams Pierre, La réaffectation des friches d'activité dans les régions de tradition industrielle. Comparaison entre l'agglomération liégeoise et le district de Sheffield

Lejeune Bernard, Analyse de la sensibilité climatique du bilan massique glaciaire dans les Alpes françaises

Lejeune Caroline, Étude de l'évolution de l'habitat en zone inondable appliquée à l'Ourthe, au Geer et à l'Eau d'Heure

Lemans Olivier, La réaffectation du bâti en zones A et B du plan d'exposition au bruit de Liège Airport. Le cas des locataires économiques

Lequeux Quentin, Évolution du littoral entre la Pointe de Champeaux et le Bec d'Andaine (Manche, France)

Lezy Thibault, Contribution des tomographies électriques et des sondages par sismique réfraction à la géographie physique

Maloir Catherine, Le nouveau port à conteneurs de Tanger Med : analyse de ses structures portuaires et étude critique de son site d'implantation. Comparaison avec les ports de Marseille et de Gênes

Marek Allyson, Analyse de la nouvelle station touristique de Saïdia (Maroc oriental) dans le cadre du développement durable

Martin Yannick, Contribution à l'identification de failles à l'activité quaternaire dans la zone de Baelen. Etude de la région de Goé - Béthane

Monseur Pierre, Contribution à l'analyse du transport en suspension de rivières de Moyenne et Haute Belgique. Application et étude spécifique d'une rivière de l'Entre Vesdre et Meuse (la Gulpe) 
511 Noël Aurélie, La palmeraie de Figuig dans le sud-est du Maroc: approche pluridisciplinaire des facteurs responsables de sa mutation Pauly Alexandra, Analyse des hausses de température nocturne à la station météorologique de Raeren Pion Geoffrey, Essai de géographie électorale. Le cas de l'extrême droite dans le Hainaut Pirard Xavier, Utilisation de la dendrochronologie en vue de mettre en évidence la dynamique de plusieurs cours d'eau du massif ardennais. Cas de l'Aisne, la Chavanne, la Lesse, la Lienne, l'Ourthe et la Wamme

Pirart François, étude à différentes échelles du réseau du commerce alimentaire en Belgique. Critères de localisation des nouveaux points de vente de proximité dans la Ville de Liège

Rixen Patrick, Étude de la localisation des agences d'intérim en Belgique. Étude à l'échelle nationale et locale

\section{Géomatique et Géométrologie}

Bovy Benoît, Étude de propagation d'erreur dans le calcul d'histogrammes bivariés pente versus altitude relative à partir d'un MNT

Broxham Kenneth, Protocole de validation d'un cas de profil géographique

Detry Geoffroy, Modélisation de la demande en transports en commun en Wallonie. Confrontation avec l'offre disponible en vue d'identifier les manquements au sein du réseau

Marcq Pierre, La promotion foncière résidentielle. Les lotissements dans la commune de Herve

Moulaert Sabine, Essai de modélisation de bâtiments : les cheminements en cas d'incendie

Quoibion Nicolas, Levé de lignes haute tension: étude de différentes méthodes et comparaisons

Willem Bernard, Analyse de données lasergrammétriques

\section{8}

\section{Géographie fondamentale}

Jacquemin Ingrid, Dynamique fluviale d'une rivière à blocs : le ruisseau du Ruaumoulin (affluent de la Semois ardennaise)

Nahon Carole, Les apports de la télédétection dans l'étude diachronique de l'envasement du barrage : le cas des retenues Mohamed V et Hassan II (bassin de la Moulouya, Maroc oriental)

Silinski Alexandra, Analyse diachronique d'une région côtière : la Mer des Wadden de la Frise du Nord (Allemagne)

Simon Florianne, Dynamique sédimentaire d'un cours d'eau corse, de la source à l'embouchure : le Golo

31 Farinalla Sophie, Géomorphologie structurale de l'Anglona (Sardaigne) par la photointerprétation. Étude de l'impact de la néotectonique sur la géomorphologie

\section{Géomatique et Géométrologie}

Graas Alexis, Développement d'outils permettant d'interpréter des données spatiotemporelles. Le cas d'une banque de données images océanographiques 

bâti

\section{climatologie}

\section{9} du Maroc) Bruxelles comportements

Henrard Jérôme, Création et analyse de qualité de Modèles Numériques de Surface et d'ortho-images créés à partir d'images CORONA

Kasprzyk Jean-Paul, Développement d'une géocodification 3D pour la modélisation du

Pezzetti Marie, Production immobilière en immeubles collectifs. Facteurs de développement et de dispersion

Dury Marie, Projections à l'échéance 2100 des changements de température et de précipitations en Belgique réalisées à l'aide des modèles du GIEC

Theunissen Yannick, Étude topoclimatologique de la partie nord-est de la carrière souterraine de Petit-Lanaye inférieure. Réflexion préliminaire à propos des conditions thermiques d'hibernation des chiroptères

\section{Géographie fondamentale}

Clerinx Bernard, Etude de dynamique fluviale. Les inversions de débit du Geer

Collard Caroline, Etude diachronique du littoral entre le Capo Negro et Ceuta (nord-ouest

Dubois Charline, La familiarité spatiale dans ses composantes cognitive et comportementale. étude de la familiarité spatiale dans les communes rurales de Bassenge et de Dalhem (Belgique)

Ledant Martin, Evaluation du potentiel de la cartographie densimétrique appliquée à des données de très grande échelle spatiale 2D et 3D. Le cas de la région liégeoise

Minet Anne-Sophie, étude de la dynamique littorale sur la côte sud-ouest du Cap Corse. La baie de Saint-Florent et les marines d'Albo et de Nonza

Onkelinx Jeremy, Ville et cinéma : des salles de quartier aux multiplexes. Analyse de trois villes: évollution spatio-temporelle et localisation des cinémas à Namur, Liège et

Plunus Julie, Paramètres hydrologiques et impacts hydrologiques et géomorphologiques des barrages de castors en région wallonne

Poncelet Nadia, Application de la transformée de Hough à la détection des linéaments et étude morphodynamique des talus d'éboulis. Recherche géomorphologique multiscalaire dans le massif de Cima Asta et la Catena dei Lagorai (Italie)

Robert Audrey, Analyse de la variabilité spatiale de la sécheresse édaphique et de ses effets sur le hêtre commun (Fagus sylvatica L.) en Région wallonne. Approche interdisciplinaire

Simon Frédéric, La localisation des commerces d'articles de sport en Belgique : essai de typologie des réseaux et de leurs modes de déploiement

Stevens Pierre, Population néerlandaise en Wallonie. Choix résidentiels et

Trotta Marie, Evolution (1979-2008) des services d'hospitalisation en Belgique. Quels liens avec la hiérarchie urbaine? 
Wallemacq Véronique, Quantification et localisation de l'érosion des berges de rivière causée par la glace de ségrégation dans un secteur à méandres de la Chavanne. Conditions sédimentologiques et topoclimatologiques

\section{Climatologie}

Belleflamme Alexandre, Etude des conditions propices aux incendies sur le plateau des Hautes-Fagnes sur base d'une classification de types de circulations atmosphériques et de réanalyses NCEP-NCAR1

Docquier David, Variabilité interannuelle de l'albédo de surface au Groenland de 1982 à 2003 à l'aide des données satellitaires AVHRR- Construction d'un masque toundra-glace annuel

Doutreloup Sébastien, Amélioration d'un modèle de prévision quart horaire des productions des parcs éoliens d'Amel et de Perwez en Belgique

Jacquemin Ingrid, Cycles à 400 ka et variabilité à plus long terme des indicateurs géochimiques au Miocène moyen : analyse à l'aide d'un modèle couplé de la géochimie et du climat.

Jorion Nicolas, Changements climatiques dans l'Oriental marocain et modification de la zone agroclimatique de Stipa tenacissima

Klein Tommy, Utilisation d'indices climatiques en vue d'analyser l'évolution des événements extrêmes de température et de précipitations en Belgique de 1958 à 2007

\section{Géomatique et Géométrologie}

Dinon Bernard, Apports des données cadastrales à l'étude du commerce de détail. Le cas des pôles périphériques secondaires liégeois

Jaspard Mathieu, Réalisation d'une application Open Source de cartographie en ligne pour la gestion des implantations commerciales

Lonchay Matthieu, Précision du positionnement par satellites. Influence de la géométrie de la constellation

Martin Francq, Liège et Limbourg, le label logistique

Saut Stéphane, Aménagement du territoire et gestion de la production immobilière résidentielle. Le cas du Grand-Duché de Luxembourg

2010

\section{Géographie fondamentale}

Beckers Arnaud, Facteurs de propagation des knickpoints dans un réseau hydrographique. Modélisation dans le bassin de l'Ourthe

Brzezinski Michaël, Cartographie et étude de l'évolution verticale récente du lit de la Hoëgne entre Royompré et Chaufheid

Denis Anne-Cécile, Étude de la dynamique de la plaine alluviale de la Lienne et des conditions de sédimentation dans la tourbière des Prés de Neucy

De Wispelaere François, Développement de l'habitat et coopération entre particuliers. Analyse comparative Wallonie - Berlin - Pays-Bas

Fabris Andréa,La localisation des agences en Belgique. Quelles logiques spatiales?

Fiebig Benoît, Les clos résidentiels fermés de Wallonie. Recensement de l'offre et évaluation contingente 
Gilson Mylène, Étude de la mise en place de la nappe de cailloutis de la Lembrée depuis la dernière glaciation

Heinesch François, S'ouvrir au phénomène de conurbation fonctionnelle. L'exemple de l'Ouest de la Province de Liège

Hussin Mélanie, Les pôles de compétitivité en Région wallonne : un outil efficace contre le sous-régionalisme?

Lesage Brice, Logiques spatiales du commerce "ethnique" à Liège

Morvan Pauline, Frontières et nouveaux développements commerciaux: quelles relations? L'exemple de la région des trois frontières

Pousseur Gilles, Les géomorphosites de Gaume. Inventaire, évaluation et projets de valorisation

Stree Julie, Les écoquartiers en Wallonie : étude de l'offre et approche de la demande

Vandermeer Caroline, Les déplacements domicile-travail en Wallonie. Le rôle de l'accessibilité et du profil des entreprises sur les répartitions modales

Vazquez Parraz Juan, Étude de l'appareil commercial wallon. Approche géométrique de la distribution spatiale de l'offre

\section{Géométrologie}

Cornet Stéphanie, Reconstitution des déplacements d'un criminel à partir de données spatio-temporelles (Erquelinnes-Maubeuge)

De Francquen Florence, Comparaison de deux solutions photogrammétriques aéroportées en vue de réaliser une cartographie à grande échelle en Antarctique

Demonceau Daniel, Prototypage d'un système d'information géographique urbain pour la gestion des risques naturels - Bamenda-ville (Cameroun)

Hansenne Clémentine, Correspondance entre relations spatiales et langage naturel : le cas du modèle de relations projectives ternaires

Lahaye Benoît, Spatiocartographie à petite échelle de la station belge Princesse Elisabeth en Antarctique

Lebbos Lucie, Développement d'opérations spatio-temporelles pour des points mobiles

Sarton Nicolas, Relevé tridimensionnel d'objets construits. Analyse de diverses techniques terrestres d'acquisition de données

Wolff Edouard, Méthodologie d'acquisition de données 3D et sémantiques à partir d'une maquette historique

\section{Climatologie}

Meurens Vincent, Sur la possibilité de modéliser l'épisode orageux du 29 mai 2008 à Liège?

Sougnez Arnaud, Caractérisation des précipitations atmosphériques sur le campus du Sart Tilman, Liège. Utilisation d'un disdromètre à laser

Strengnart Florian, Analyse climatique et amélioration des prévisions de brouillard à l'aéroport de Charleroi- Bruxelles Sud

2011

Géographie fondamentale 
Ansay Robin, Les parcs scientifiques wallons. Quel impact sur le redéploiement régional ?

Barbette Céline, Parcs culturels : Outils de développement régional ou outils de protection du patrimoine

Beghin Jérémie, Étude paléoclimatique multi-proxies lors des épisodes de glissements de terrain holocènes du Pays de Herve

Blaffart Maud, Analyse de l'application, de la pratique et de la mise en oeuvre du schéma de structure communal. L'aménagement stratégique au niveau communal

Borgoens Céline, Analyse du nombre d'arrêtés "calamité inondation" par commune : les risques réellement encourus. Application en Région wallonne

César Émilie, Modelling the hydrological response of an urban watershed. Diachronic analysis of the effects of land use changes on storm runoff generation in the Upper Bukit Timah basin, Singapore

Condé Gilles, Mutations du système de villes belge. Évolution économique des régions urbaines entre 1981 et 2006

Detienne Marie, Impacts des dépôts de cendres volcaniques sur les propriétés physiques des sols : Expériences en laboratoire et modélisation. Cas d'un andosol brun islandais

Dimitriadis Nathanaël, La consommation d'espace par les activités dans les zones d'activités économiques. Comparaison entre le Grand-Duché de Luxembourg et la province du Luxembourg

Dupaix Thomas, Les logiques de localisation des centres de remise en forme wallons

Gilson Martin, La localisation des maisons de repos en Belgique. Quelles logiques spatiales?

Gischer Loïc, Le soutien des débits en période d'étiage. Exemples pris en Wallonie

Leroy Guillaume, Le RAVeL, un nouveau point de vue sur le territoire. Etude de la perception, de l'appropriation et de la fréquentation d'une voie verte par ses riverains et usagers, le cas du Réseau Autonome de Voies Lentes (RAVeL) de Charleroi et Liège

Libert Virginie, Repopulation des villes. Étude de cas : Région de Bruxelles-Capitale, Ville de Liège et Ville de Gand

Loiseau Véronique, Toits verts et rénovation urbaine: monétarisation et internationalisation de l'externalité paysagère. Application de l'évaluation contingente dans le quartier Saint-Léonard à Liège

Strivay Nicolas, La sédimentation dans la retenue de Robertville. Estimation du taux de dénudation du bassin versant de la Warche et modalités de la répartition spatiale des sédiments au sein de la retenue

Weber Lidvine, Apport des données cadastrales relatives à l'âge des bâtiments à la géographie urbaine. Analyse de l'occupation de l'espace et des noyaux d'habitat

\section{Géomatique et Géométrologie}

Begon Jean-Michel, Prototypage d'un serveur de données géographiques maillées: Rasdaman

Garwig Kevin, Prototypage d'une architecture spatiale OLAP pour l'intégration et l'analyse en ligne de données démographiques et relatives aux infractions

Guérard Arnaud, Correction de séries temporelles d'images classifiées sur base de l'analyse de la rationalité des trajectoires de changement 


\section{Climatologie}

Bader Whitney, Extension of the long-term total column time series of atmospheric methane above the Jungfraujoch station: analysis of grating infrared spectra between 1977 and 1989

Grignet Yannick, Approche hédonique du marché immobilier sur base d'annonces internet : les maisons d'habitation au sein de la région urbaine de Liège

Iniguez Hervé, Influence de la nouvelle génération de station totale sur les méthodes de géocodification 3D

Jonlet Benoît, Influence des nouvelles générations d'équipements topographiques sur les méthodes de levés souterrains

Lang Charlotte, Modeling of the surface mass balance in Svalbard with the regional climate model MAR over 1958-2010

Leclercq Luc, Aide à la navigation intérieure. Le franchissement des obstacles

Marchal Gilles, Simulation des flux de trafic au travers des systèmes multi-agents

Mokas Amalia, Modélisation des glaciations globales du Néoprotérozoïque : influence de la vitesse de rotation de la Terre sur l'entrée en glaciation

Sacré Bernard, Évaluation du modèle régional du climat WRF au Groenland

\section{- Université Libre de bruxelles}

2007

\section{Géographie humaine}

Babar Louise, Analyse socio-spatiale de différents types de pauvreté à Nankin

Bonami Déborah, L'évolution des caractéristiques des habitants des quartiers de logements sociaux à Bruxelles

Castellano Nicolas, Bruxelles: logiques de localisation des Italiens et de leurs infrastructures (restaurants)

Courtois Xavier, Géographie de la vitesse commerciale sur le réseau de la STIB

Dufermont Emilie, L'aménagement des espaces publics à Liège

Dupuis David et Flament Sarah, Evolution récente d'un front de colonisation agricole du sud-ouest Niger dans un contexte de saturation des terroirs: stratégies d'adaptation paysannes

Dupuis Aurélien, Carrières résidentielles des Marocains à Bruxelles

Hanson Emilie, Analyse visuelle et typologie de la morphologie des quartiers à partir d'une image à très haute résolution spatiale : le cas de Bagdad (Irak)

Hertsens Claire, Analyse de la répartition géographique des organisations non gouvernementales belges francophones dans les pays du Tiers Monde

Micheroux Nicolas, Vivre pauvre au centre ou en banlieue : avantages et désavantages d'une localisation centrale pour les populations défavorisées

Quittelier Benoit, Effets des politiques d'importation de l'Union Européenne sur la culture de la banane : comparaison du Costa Rica et de la Martinique 
Rutaganda Tatien, Etude de la gestion spatiale des déchets ménagers solides dans l'entité de Mons

Ubieta Céline, Diffusion spatiale à l'échelle mondiale des parcs d'attraction à thème

Diplôme d'Etudes approfondies DEA

Kayembe wa Kayembe Mathieu, La périurbanisation en Afrique subsaharienne : les cas de Cotonou et de Kinshasa

\section{Géographie physique}

Calvi Benoit, La fragmentation de l'habitat de la mouche tsé-tsé

Carnat Gauthier, Contrôles physico-chimiques sur les teneurs en dms, dmsp et dmso de la glace de banquise printanière de l'océan austral (Arise Cruise, Casey station, septembreoctobre 2003) : implications climatiques

De Bellefroid Augustin, Elaboration d'un itinéraire touristico-scientifique sur le thème du karst, sur le plateau de Padis-cetatile Ponorului, Monts Apuseni, Roumanie

Duhannoy Dimitri, Le Kraski Rob (Slovénie) et ses escarpements: synthèse multidisciplinaire et "itinéraire pédagogique"

Festraets Valérie, Détection automatique des haies et des arbres isolés sur des images satellitaires à très haute résolution spatiale en relation avec les mesures agrienvironnementales de la politique agricole commune

Goossens Thomas, Contrôles physico-chimiques sur les teneurs en dms, dmsp et dmso de la glace de banquise estivale de l'océan austral (Ispol Cruise, Western Weddell Sea, décembre 2004) : implications climatiques

Lejoly Florence, Typologie et géographie des campings wallons en fonction des risques potentiels qu'ils présentent pour l'environnement (étude de cas: la province de Luxembourg)

Van den Broeck Jean, Détection et segmentation sur des images satellitaires à très haute résolution spatiale des parcelles incluant des surfaces non agricoles

Van Overmeiren Gaëtan, Etude des caractéristiques aquatiques du site du "confluent" à Rixensart

2008

\section{Géographie humaine}

Boka G., La diffusion spatiale du mouvement punk en Belgique francophone

Debroe E., L'urbanisme défensif à Molenbeek-Saint-Jean

Gilles de Pelichy A.C., Cartographie du risque de dégâts aux cultures causés par les grands animaux dans la West Patauke Game Management Area en Zambie

Hervo M., La hiérarchie commerciale des communes françaises : essai de détermination et analyse sur base du commerce de détail

4 Lothe A., Impacts des changements climatiques sur le mode de vie des populations locales en Alaska : le cas d'Huslia

Pion G., Géographie électorale du non-vote et de l'extrême droite en Wallonie : une étude de cas au niveau régional et infra-communal

Pirard A., La propagation de la grippe aviaire en relation avec la géographie économique de la filière avicole 
2009 Kimbanseke numériques Région wallonne

Vivegnis L., Géographie des dégâts aux cultures occasionnés par certains mammifères, appliquée dans un modèle zambien de gestion de la faune sauvage

\section{Géographie physique}

Dartois Maxime, Etude comparative de fleuves littoraux bretons. Influences de l'eutrophisation et des impacts anthropogènes

Van Humbeeck Felix, Contribution à l'analyse des glaces situées à la base de la calotte de Akademii Nauk, archipel de Severnaya Zemlya, Arctique russe

\section{Géographie humaine}

Biernaux M., Arctique et réchauffement climatique, approche systémique d'une situation géopolitique en pleine évolution

Billotte A., Formes exogènes du maraîchage autour de Niamey

Chevalier C., Modélisation du transport de marchandises en Europe : Analyse critique et applications statistiques sur la matrice origine-destination WORLNET 2005

Duplat A., L'agriculture urbaine et périurbaine face aux contraintes foncières à Kinshasa (RDC) : quelle(s) solution(s) pour une activité durable? Cas du site maraîcher de

Henry A., Les inégalités socio-spatiales d'accès aux services urbains dans les pays en développement : le cas de l'enlèvement des ordures ménagères à Abidjan

Wéron B., L'offre touristique dans le Benelux : cartographie de synthèse

Wertz I., La réorganisation spatiale de la Slovénie suite à la dissolution de la Yougoslavie jusqu'à son entrée dans l'Union Européenne - Analyse au niveau communal

\section{Géographie physique}

Bastin David, Premières mesures de DMSO dans la glace de mer antarctique (ISPOL Cruise, Western Weddell Sea, décembre 2004) : implications pour l'impact du cycle du soufre sur les changements climatiques

Coppens Sarah, Contribution à l'étude des gaz à impact climatique dans la glace de lac en région périglaciaire : caractérisation du contenu total et des compositions en gaz pour différents types de glace

Defrance Dimitri, Modélisation de la ligne d'ancrage et des calottes marines : amélioration de la représentation des processus de migration de la ligne d'ancrage dans les modèles

Deneubourg Milène, Premières mesures du DMSO (oxyde de dimethylsulfure) dans la glace de mer antarctique printanière (secteur australien) : implications pour l'impact du cycle du soufre sur les changements du climat

Depoorter Mathieu, Contribution à l'étude des gaz à impact climatique dans la glace de lac en région périglaciaire : étude comparative du contenu total et des compositions en gaz dans 4 lacs scandinaves

Dewitte Ben, Méthodologie de gestion de la qualité des eaux de baignade. Etat des lieux en

Fostier Nicolas, Détection des eaux de surface près du Parc Kruger (Afrique du Sud) : transfert d'échelle de la très haute à la haute résolution spatiale 
Julémont Christophe, Analyse des variables sensibles et des implications géopolitiques dans la gestion des ressources hydriques de la ville de La Paz - El Alto

Moreels Sandra, Développement de la géochimie d'éléments traces et majeurs en tourbière comme traceurs des flux et des sources des dépôts de poussières durant l'Holocène : la tourbière du Pinet dans les Pyrénées françaises

Nuttin Laurence, Enregistrement des changements environnementaux dans les sédiments lacustres chiliens $\left(45^{\circ}-48^{\circ} \mathrm{S}\right)$ au cours des derniers millénaires

\section{0}

\section{Géographie humaine}

Belffort G., Chronologie comparée du développement du commerce moderne dans les différents pays européens et les facteurs de ces différences de chronologie, en particulier : évolution du pouvoir d'achat, des taux d'équipement des ménages et de la législation sur le grand commerce

Benameur H., L'enseignement de la géographie confronté à la perception spatiale des élèves en fin de premier degré: étude de cas effectuée dans l'enseignement de la Communauté française de Belgique

De Spiegeleire Y. et Evens M., "Les effets structurants" du tramway en France : Etude de cas de la ville de Strasbourg et de Montpellier

Destrument L., Carrières résidentielles et appropriation de l'espace par les membres de la grande bourgeoisie résidant en région bruxelloise et à ses alentours: approche individuelle

De Montpellier C., Localisation, analyse et estimation des effectifs de population via la télédétection en bordure du parc Kruger, Afrique du Sud

Dierge A., Optimisation du choix de zones adaptées à l'agriculture périurbaine de basfond par l'utilisation de données spatiales et socio-économiques

Dirckx T., Adaptative reuse in Brussels and Copenhagen: the capacity of the existing building stock to meet new functional needs

Dumont G., Géographie des projets immobiliers résidentiels récents dans les quartiers anciens bruxellois

Genart F., Géographie et impacts des mouvements de jeunesse durant la période estivale

Larsimont R., Interaction entre les changements dans l'occupation du sol et la surexploitation des écosystèmes aquatiques en Castilla-La Mancha

Lebrun K., Les nouvelles gares urbaines Opportunités à Bruxelles dans le cadre du R.E.R.

Maghouz S., Typologie des plans historiques des villes en Europe

Piraux J., Factors influencing the development of the urban cycling. A case study of three European cities : Brussels, Copenhagen and Vienna

Rome E., Formation de l'espace touristique balnéaire en Turquie

Schneider G., Diffusion de la fièvre aphteuse: Analyse paysagère des possibilités de contacts entre les buffles et le bétail, Parc National Kruger, Afrique du Sud

Schools S., Enseigner la géographie: des méthodes distinctes pour des publics différenciés ? Etude de cas en région bruxelloise 
Wyn B., Comparaison de la télédétection et d'une enquête de perception comme outils d'analyse de l'érosion dans les Andes Application à la région de Santa Ana, Cuenca, Equateur

\section{Géographie physique}

Callens Denis, Variabilité spatiale du bilan de masse du FranKenny Ice Rise, Dronning Maud Land, Antarctique

De Greef Grégory, Modélisation de l'évolution historique et récente du glacier Chacaltaya dans les Andes boliviennes

Gilson Gaëlle, Composition en DMS et composés soufrés associés de la glace de mer annuelle arctique en Mer de Beaufort (CFL, Canada) et implication pour les flux de DMS vers l'atmosphère

4 Hamoir Marie-Noëlle, Rôle de la morphodynamique fluviale sur les flux de matières en suspension: le facteur de contrôle géomorphologique $\mathrm{m}$ constitue-t-il un outil méthodologique de choix?

Loriaux Thomas, Estimation du volume de deux glaciers via radio-écho sondage et relevés GPS, Cordillère Royale, Bolivie

Visart de Bocarme Moïra, Influence de la température et de la $\mathrm{pCO} 2$ sur les coccolithophores

Willame Alan, Premières mesures à haute résolution de la concentration en dioxyde de carbone dans la glace de mer : un exemple arctique, la mission Barrow (janvier-juin 2009) - implications pour les flux du $\mathrm{CO} 2$ en régions polaires

Zhou Jiayun, Evolution des propriétés biogéochimiques des glaces de mer annuelles côtières (Barrow, Alaska) : implications pour la productivité primaire et les flux de gaz à effet climatique

\section{1}

\section{Géographie humaine}

Bricmont Antoine, Approche géographique de la financiarisation de l'immobilier à travers le cas des sicafi et du marché de bureaux

Choteau Alexis, Périurbanisation de l'emploi et accès au marché du travail. L'exemple de trois villes: Bruxelles, Anvers et Liège

Dehouck Simon, Stratégies globales de localisation des compagnies pétrolières dans le secteur de la production et du raffinage

De Leeuw Christophe, Le renouveau du tramway en France : type de population desservie par ces nouveaux tramways

Dieudonné Régis, Localisations et périurbanisations des activités de bureaux dans la métropole bruxelloise sur la période 2000-2010

Federighi Nicola, Analyse multiscalaire de la diffusion de la salsa et du tango argentin

Libert Mathieu, Les dynamiques socio-économiques le long de l'axe Namur-Luxembourg

Oulad Chaikh Mohammed, Logiques contemporaines de développement industriel dans la région de Tanger-Tétouan

Veiders Caroline, Les cimetières dans les plans urbains à partir de la fin du $18^{\mathrm{e}}$ siècle 
Volpagni Damien, Fonctionnement et motivation des migrants à destination de la Province de Luxembourg

\section{Géographie physique}

Camelbeeck Mathieu, Modélisation de l'impact des propriétés du sol et de l'érosion à l'échelle d'un bassin

Crabeck Odile, Etudes des processus physico-chimiques affectant la teneur et la composition des glaces de mer de Gothabsfjord (Nuuk, Groenland) et relations avec les flux de $\mathrm{CO} 2$ à l'interface glace-air

Deleu France, Etudes des propriétés physico-chimiques des glaces de mer artificielles de l'expérience européenne INTERICE IV et relations entre concentrations en CO2 dans la glace et flux glace-air de $\mathrm{CO} 2$

Flament François, Analyse des propriétés physico-chimiques et cristallographiques des glaces basales du sondage antarctique de Bekner Island (Ronne et Filchner Ice shelves)

Godefroid Hugues, Etude thermique de l'éruption du Rinjani (mai 2009) par les données satellites MODIS

Maertens Anaëlle, Etude cinétique de la dissolution de carbonates de calcium dans l'eau de mer

Rans Guillaume, Etude sur l'efficacité des barrières végétales et naturelles à protéger les côtes du Sri Lanka à l'encontre des vagues maritimes d'évènements naturels extrêmes

Van Liefferinge Brice, Conditions intraglaciaires du glacier McCall, USA

\section{- Universiteit Antwerpen}

\section{7}

\section{Transport and Regional Economics}

Ceuppens Annelies, Het economisch belang van transshipment in de Vlaamse zeehavens

Claessens Bart, Shortsea shipping in de haven van Antwerpen. Onderzoek naar de uitdagingen en ontwikkelingen

Devolder Herwig, Toekomstperspectief van de Duitse zeehavens in Noord-West-Europa

De Neve Bram, Inland containerterminals : vergelijkende studie tussen vlaanderen en Nederland

De Nys Yves, Het economisch belang van city-airports voor de stedelijke economie

Dielen Dennis, Kostenvoordelen verbonden aan clustervorming

Haerens Friso, De logistieke aantrekkelijkheid van de regio Zuid-West-Vlaanderen

Herrebosch Matthias, Een gemeenschappelijk zeehavenbeleid voor Antwerpen en Rotterdam : opportuniteit of kannibalisme?

Kupfer Franziska, Luchthaveneilanden - een oplossing voor de toekomst ?

Leyssen Willem, Knelpunten voor de Antwerpse Haven

Sauviller Pieter, Parkeergebouwen in België : lessen uit de Verenigde Staten

Schandevyl Bert, De invloed van het Panamakanaal op de containerlijnvaart

Smeuninx An, Criteria voor de vestiging van logistieke bedrijven binnen versus buiten het havengebied 
Van Daele Pieter-Jan, De Vlaamse beleidsnota's over de zeehavens: vijf zielen, één gedachte?

Van de Leur Philip, De rol van inlandterminals in de logistieke keten

Vanholme Thomas, Analyse van de logistiek in Wallonië

Van Impe Kathleen, Identificatie van de containerstromen tussen de havens en de multimodale terminals in Vlaanderen

Verberght Edwin, Shortsea shipping en binnenvaart in de Europese Unie

Verdickt Andreas, Containerbinnenvaartterminals : toekomstperspectieven binnen een groeiende markt

Wattelé Rob, De evolutie van bottlenecks op het Vlaamse wegennet

Zwaans Jill, Gevolgen van de verbreding van het Panamakanaal voor de maritieme sector

2008

\section{Transport and Regional Economics}

Braat Johan, Het effect van industrialisatie op havenactiviteiten

Callens Bart, De ontbanking van België : een economisch geografische analyse

Choukri Abdelmajid, Marokaanse zeehavens: sterkten en zwakten van havens aan de Atlantische Oceaan versus Middellandse zee

Druyts Bert, Analyse van de Oost-Westontsluiting rond Mechelen

El Hattach Yassine, De impact van het Tanger-Med project op de Marokkaanse economie

Heymans Nicolas, De modal ontsluiting van de haven van Antwerpen, analyse van bedrijfsbeslissingen

Peeters Tine, Havenconcurrentie op de markt van het niet-gecontaineriseerd vervoer

Rosseel Caroline, De concurrentiële positie van de haven van Zeebrugge naar het hinterland

Van Deun Kathleen, Worden grote treinstations aantrekkelijke shoppingcenters?

Van Hecke Koen, Toheffing in Duitsland : analyse van effecten

Van Hulle Frederik, Vergelijkende studie in de aanlooopkosten tussen Antwerpen en Rotterdam (shortsea shipping)

Van Zeebroeck An, Reürbanisatie in Vlaamse steden

Vissers Jurgen, Masterplan Antwerpen, een evaluatie

2009

\section{Transport and Regional Economics}

Braspennincx Dieter, De haven als knooppunt van verschillende transportmodi in de logistieke keten

Cant Jeroen, Het Saeftinghedok : economische noodzaak of mythe

Colbrant Charlotte, Het effect van lowcost luchtvaartmaatschappij op jongerenmobiliteit

Genbrugge An, Rekeningrijden, de oplossing voor het fileprobleem?

Hens Dennis, Vergelijking van de luchtvaartstrategieën van luchthavenbeheerders

Maes Bénédicte, Uitbouw van een haven : vergelijking Shanghai-Antwerpen

Nuyts Manu, Invloed van filevorming op transport- en logistieke beslissingen in bedrijven 
Sarrouj Junis, Economische en ruimtelijke analyse van Dubai Maritime City (duo met Lorenzo Simons)

Struyf Els, Factory outlets : economisch-geografische analyse

Van de Schoor Kim, Het economisch belang van de Yzeren Rijn

Van Hove Dennis, World Maritime Cities : een update

Verstappen Pieter, Analyse van filevorming op het wegennet

2010

\section{Transport and Regional Economics}

Blomme Nele, De logistieke organisatie van ontwikkelingssamenwerking

Collier Willa, Vlaamse stadsvernieuwingsprojecten: evolutie van de vastgoedprijzen in hun omgeving

De Vlieghere Stéphanie, De competitie tussen lagekostenluchtvaartmaatschappijen

Jonckheere Jeroen, Vegerlijkende studie van stedelijke fietsverhuursystemen

Smout Koen, De gevolgen van de spoorliberalisering: nieuwe producten en nieuwe spelers

Van Reusel Dennis, Mogelijke gevolgen van een Noord-Zuid verbinding in de Kempen

Yskout Yves, Concurrentie tussen low-cost carriers : een case studie analyse

2011

\section{Transport and Regional Economics}

Flies Lucas, Plaatsvestigingsonderzoek Gamma

Helsen Marie, De in- en uittrede in de sector van het zeevervoer

Janssens Jonas, Rankings van steden : kritische vergelijking van resultaten van vastgoed actoren en academische studies

Jochems Leen, Verplaatsingsgedrag van studenten naar de Antwerpse universiteit/ hogeschool

Leemans Pieterjan, Nachtvervoer : Toepasbaar in alle sectoren?

Stevens Laurence, Grensoverschrijdende samenwerking tussen havens

Stevens Tinne, Economische analyse van de strategie van luchtvaartmaatschappijen uit het Midden-Oosten

Vandenbroeck Carl, Economische analyse van het Trans Europees Netwerk concept

Vangramberen Charlotte, Spoorvrachtvervoer in Europa: domineert Deutsche Bahn de markt?

Vossen Niels, Perifere P\&R zones: een oplossing voor verkeerscongestie in de binnenstad?

Wellemans Anneleen, "Hinterworld" van Vlaamse zeehavens

\section{- Vrije Universiteit Brussel}

2007

Fysisch Milieu 


\section{Sociaal Milieu} Centrum

GIS

2009

2010

\section{Fysisch Milieu}

De Haeck Sebastiaan, Numerieke modellering van de frontvariaties van de Morteratschgletsjer, Zwitserland

Denys Sarah, De contrasterende Holocene sediment successie langsheen het westelijk en oostelijk deel van de Belgische kust : oorzaak en gevolgen

Quisthoudt Katrien, Mangrove en garnalenkweek in de Lagune van Chilaw (Sri Lanka)

Toestand en perspectieven verlaten garnaalkwekerijen

Van Ophem Jeremy, Bepaling van de massabalanskarakteristieken van de Morteratschgletsjer, Zwitserland, aan de hand van satellietbeelden

Colpaert Lien, Een onderzoek naar de sociale geografie van de Chinese gemeenschap in het Brussels Hoofdstedelijk Gewest

Lauwers Liza, Verhuiseffecten van stadsontwikkeling in Antwerpen. Een kwantitatief onderzoek naar intrastedelijke migratiepatronen

Robyns Olivier, De bereikbaarheid van formele en populaire vrije tijd infrastructuren voor jongeren binnen het Brussels Hoofdstedelijk Gewest

Van Hamme David, Globalization from below : De Brabantstraat : a space of global flows ?

Vermeulen Sofie, Een onderzoek naar de utopie en hedendaagse stadsprojecten in Brussel. Cases : de Zinneke Parade, kunstencentrum WIELS en het Rijksadministratief

Dujardin Rutger, Remote sensing study of a possible impact crater : The Luizi structure, Katanga, Congo Democratic Republic

Vanderhaegen Sven, Karakterisering van stedelijke morfologie aan de hand van remote sensing data, toegepast op Brussel

Vangeneugden Rob, Een GIS-model voor het intermodaal terminallandschap in België : Opbouw en simulaties

Vlaeminck Jeroen, Kartering van stedelijk groen in Brussel en omgeving aan de hand van Landsat ETM+ data

Langenus Kelly, Cork city : een case study van het Ierse succesverhaal

Vankeirsbilck Céline, Typering van de karakteristieken van de stedelijke leefomgeving op buurtniveau in Brussel en omgeving

Van Mulders Irina, Analyse van de evolutie van de groene ruimte in Brussel en de Brusselse rand (1978-2001) aan de hand van medium-resolutie satellietdata

Beke Elise, Invloed van de ouderdom van ontbossing op de opslagvan bodemorganische koolstof in Midden-België

De Troch Rozemien, De relatie tussen meteorologische condities en bosbranden in Griekenland, Portugal en de Canarische Eilanden : een vergelijkende studie 
De Vleeshouwer David, Time-series analysis on selected geological sections. A search for cyclicity

1 Jolley Robin, Holocene fluvial-aeolian interactions in the western Nile valley, Upper-Egypt Sauvage Justine, Distribution of Platinum Group Elements across the Cretaceous-Tertiary Boundary, Denver Basin (Colorado)

Tassignon Steven, Invloed van erosie op organische koolstof diepte verdeling langsheen een helling onder akkerland en weiland in de Belgische Leemstreek (Herzele)

Van der Stocken Tom, Determination of the routing of basal water under the Antarctic ice sheet and its meaning in het formation of subglacial lakes and the processes of basal sliding

\section{Sociaal Milieu}

Dony Colline, Sustainable development of Brussels in a global-local nexus. Local effects of a global agenda 21

GIS

Crols Tomas, Ontwikkeling van optimale equivalente kaartprojecties voor globale toepassingen

2011

\section{Fysisch Milieu}

Geeraert Naomi, Modelling the front variations of the Morteratsch glacier, Switzerland

Jacobs Bram, The Zwin as a tidal channel evolution throughout the Holocene

Zekollari Harry, Modelling the dynamics of the Morteratsch glacier complex (Engadin, Switzerland) using a three-dimensional higher-order flow model

\section{Sociaal Milieu}

De Luyck Niels, Gentrificatie in Brussel : komen buurten onder druk te staan? Case study Dansaert en historisch Molenbeek

Matriche Lauréline, Een tijdgeografie van EU-expats in Brussel

Mommens Koen, Etnisch ondernemerschap in gentrifiërend historisch Molenbeek

Starbuck Stephen, Spanningsbuurten: een gezonde sociale mix en/of buurten onder druk? Casestudie op 13 Anderlechtse statistische sectoren

GIS

De Crem Aloïs, Ruimtelijke analyse omtrent de evolutie van bereikbaarheid van postale diensten aangeboden door bpost aan particulieren in Vlaanderen

De Vuyst Karel, Analyse van de verstedelijkingsdynamiek in en rond het Brussels hoofdstedelijk gewest aan de hand van medium resolutie satellietdata (1978-2001) 